\title{
KISS: A NEW DIGITAL SURVEY FOR
}

\section{EMISSION-LINE OBJECTS}

\author{
A. KNIAZEV ${ }^{1}$, J. SALZER ${ }^{2}$, V. LIPOVETSKY ${ }^{1}$, T. BOROSON ${ }^{3}$, \\ J. MOODY ${ }^{4}$, T. THUAN ${ }^{5}$, Yu. IZOTOV ${ }^{6}$, J. HERRERO ${ }^{2,7}$ AND \\ L. FRATTARE ${ }^{2,8}$ \\ ${ }^{1}$ Special Astrophysical Observatory, Russia \\ 2 Wesleyan University, USA \\ 3 NOAO, Gemini Project, USA \\ ${ }^{4}$ Brigham Young University, USA \\ ${ }^{5}$ University of Virginia, USA \\ ${ }^{6}$ Main Astronomical Observatory, Ukraine \\ 7 Center for Astrophysics, USA \\ ${ }^{8}$ Space Telescope Science Institute, USA
}

\section{Introduction}

We have initiated a major new survey for emission-line galaxies (ELGs) which we call the KPNO International Spectroscopic Survey (KISS). Survey observations began in March 1994 with the 0.61-m Burrell Schmidt telescope ${ }^{1}$. The technique we employ combines the benefits of a traditional photographic objective-prism survey with the advantages of using a CCD detector. The field of view of our CCD is $1.1^{\circ}$ square, and the prism employed provides a dispersion of $19 \AA /$ pixel at $5000 \AA$. The spectral range covered $(4800-5500 \AA)$ is restricted by a specially designed filter that transmits from rest-frame $\mathrm{H} \beta$ to just shortward of the strong night-sky line at $5577 \AA$; this greatly reduces the sky background. We expect KISS to be sensitive to galaxies with magnitudes as faint as $\mathrm{B}=20^{m}-21^{m}$, much deeper than existing photographic surveys. Our initial pilot project covers 100 square degrees (Salzer et al. 1994) and overlaps the CfA/Dartmouth Century Redshift Survey $\left(\alpha=8^{h} 30^{m}-16^{h} 45^{m}\right.$ and $\left.\delta=29^{\circ}-30^{\circ}\right)$ in the North Galactic cap.

\footnotetext{
${ }^{1}$ Observations made with the Burrell Schmidt of the Warner and Swasey Observatory, Case Western Reserve University.
} 
We are able to detect in our survey: galaxies with redshifts up to $\mathrm{z}=0.10$ showing strong [OIII] $\lambda 5007$ line, high redshift galaxies via their [OII] $\lambda 3727$ emission in the range $\mathrm{z}=0.23-0.48$, QSOs with redshifts in the range $2.9-3.5$ via $\operatorname{Ly} \alpha$.

Among the goals of the survey are: obtain a deep sample of ELGs, complete to 2-3 magnitudes fainter than previous surveys; study the large-scale distribution of emission-line galaxies and their relation with normal galaxies; search for new extremely low metallicity dwarf galaxies like I Zw 18 or SBS 0335-052; determine the luminosity function for star-forming galaxies, with special emphasis on the faint end.

The initial strip has been completely observed and partly processed. Follow-up spectra of some newly discovered candidates have been obtained with the Russian 6-m telescope. Future plans include: (1) survey additional areas of the sky; (2) obtain follow-up optical spectroscopy of all candidates with a multifiber spectrograph like HYDRA; (3) obtain objective-prism images in other spectral ranges (e.g., around $\mathrm{H} \alpha$ ); (4) application of our survey method to larger telescopes.

\section{Survey Data Reduction and Some Preliminary Results}

The survey input data consist of deep direct images taken using two filters, $\mathrm{B}$ and $\mathrm{V}$, images with low-resolution prism spectra and short exposure direct $\mathrm{B}$ and $\mathrm{V}$ images taken on photometric nights in order to calibrate the magnitudes of the sources.

For the reduction of this complicated data set new software was developed in both the MIDAS and IRAF environments. The results presented here were obtained with the former package, developed principally by AK. The reduction procedure consists of the following steps: (1) preliminary reductions, (2) alignment and combining of direct and spectral images, (3) 2D sky fitting (Shergin et al. 1996), (4) adaptive filtration for the direct images, (5) transformation of the direct image positions to the spectral image, (6) direct image inventory, (7) astrometry using the direct images, (8) B and $\mathrm{V}$ photometry using the direct images, (9) $1 \mathrm{D}$ spectra extraction, (10) searching for emission features in spectra, (11) detection and tabulation of candidates with emission features.

The Output KISS Database contains accurate positions (X, Y, $\alpha(1950)$, $\delta(1950)$ ), magnitudes, colors and Star/Galaxy classification for all objects found during the inventory of the combined direct image plus additional information (position of emission peak, signal-to-noise ratio (SNR) for that peak, etc.) for all objects detected in the spectral image. In addition, the Database contains 1D extracted spectra, brightness profiles in the spatial 
direction, noise vectors (noise value for each pixel of spectrum), and spectra of continuum and continuum subtracted spectra for all extracted objects.

All ELG candidates selected in each KISS field are copied into a separate Output KISS Database. Typically 40-50 candidates are selected in each field. Final inspection of selected candidates above the threshold SNR value is made visually. It allows us to remove all complicated cases (artifacts, patterns of saturated bright stars, overlapping spectra, etc.) which cannot be filtered out with our current algorithms.

To estimate the quality of our photometry, astrometry and Star/Galaxy classification we compare the results of our reductions with results from Willmer et al. (1996), Moreau et al. (1995) and data from the APM machine. For this comparison we have identified all objects from those papers which have counterparts in the KISS fields $\alpha=12^{h} 55^{m}, 13^{h} 00^{m}$, $13^{h} 05^{m}, 13^{h} 10^{m}$ and have examined the differences in the photometry and positions of these objects between the mentioned papers and the KISS derived values. We summarize the results of this comparison below.

\section{Conclusions}

1. A new method of automatic search for emission-line candidates has been developed and applied to our deep survey data.

2. The new software for the search for emission-line candidates has been written in both the MIDAS and IRAF environments.

3. The comparison of our data with other surveys shows:

(a) In spite of our data undersampling $\left(1\right.$ pixel $\left.=2.07^{\prime \prime}\right)$ the astrometric precision after reduction is about $0.5^{\prime \prime}$, good enough for followup spectroscopy of candidates with a multifiber spectrograph like HYDRA.

(b) KISS has reliable photometry to at least $\mathrm{V}=18.5^{m}$ when compared to these other surveys (absolute error is $\leq 0.15 \mathrm{mag}$.). Internal photometric errors are $\leq 0.10$ mag. at $\mathrm{V}=20^{m}$.

4. The follow-up spectroscopy with the 6 -m telescope proved that there is no noticeable bias between candidates selected visually and automatically. Candidates above the SNR threshold are shown to be real emission-line objects of various types.

\section{References}

Moreau O. and Reboul H., 1995. Astron. Astrophys. Suppl., 111, 169

Salzer, J., et al. B.A.A.S., 26, 916

Shergin, V.S., Kniazev A.Y. and Lipovetsky, V.A. 1996. Astr. Nachrichten, 2, 95

Willmer, C.N.A., et al. Astrophys. J. Suppl., 104, 199 